\title{
Integración sensorial y trastornos de aprendizaje del código lectoescrito ${ }^{1}$
}

\section{Sensory integration and learning disorders of the literacy code}

\section{Integração sensorial e distúrbios de aprendizagem do código de alfabetização}

Olga Lucía Agudelo Valdeleón², Gloria Denyce Martín Parada³, Ada Ivonne Rojas Rojas ${ }^{4}$, Olga Lucía Torrijos Rivera ${ }^{5}$, Rolan Arcadio Correa López ${ }^{6}$

Fecha de recepción: 08/10/2018

Fecha de evaluación: 10/11/2018

Fecha de aceptación: 20/11/2018

Disponible en línea:

DOI: https://doi.org/10.18359/reds.4358

Cómo citar este artículo:

Agudelo, O. L., Martín, G. D., Rojas, A. I., Torrijos, O. L. y Correa R. A. (2018). Integración sensorial y trastornos de aprendizaje del código lectoescrito. Revista Educación y Desarrollo Social, 12(2), 33-52.

\footnotetext{
1 Los autores redactaron este texto como estudiantes de la Especialización en Neuropsicopedagogía Infantil de la Universidad Católica Luis Amigó, a excepción de Rolan Arcadio Correa López, docente asesor.

$2 \quad$ Psicóloga. Correo electrónico: olga.agudeloal@amigo.edu.co Fonoaudióloga. Correo electrónico: gloria.martinpa@amigo.edu.co Licenciada en Lingüística y Literatura. Correo electrónico: ada.rojasro@amigo.edu.co Psicóloga. Correo electrónico: olga.torrijosri@amigo.edu.co

6 Magíster en Neuropsicología Clínica. Correo electrónico: rolan.correalo@amigo.edu.co
} 


\title{
Resumen
}

La integración sensorial es la capacidad del cerebro para integrar y procesar de manera adecuada los estímulos que se reciben del entorno y generar las respuestas apropiadas según el momento determinado. Por eso cuando la integración sensorial no funciona de manera adecuada, es ineficiente o irregular, la vida de un individuo se puede ver afectada de manera significativa. Dichas dificultades se evidencian de manera más detallada en el inicio de la vida escolar del niño, pues es cuando se dan los procesos lectográficos. Es por esta razón que el objetivo del presente artículo es abordar y explicar la relación de la integración sensorial con los trastornos del aprendizaje —y más específicamente del código lectoescrito- y su importancia dentro del proceso de enseñanza. Una revisión de diferentes investigaciones científicas en el ámbito internacional y nacional evidencia que un déficit en el procesamiento sensorial supone un alto porcentaje de influencia negativa en los procesos cognitivos que permiten llevar a feliz término un aprendizaje significativo.

Palabras clave: código lectográfico; integración sensorial; trastornos del aprendizaje.

\begin{abstract}
Sensory integration is the ability of the brain to integrate and process in an appropriate way those stimuli that are received from the environment and generate the appropriate responses that are required at a certain moment; that is why, when this sensory integration is given in an inadequate, inefficient or irregular manner, it can significantly affect the life of an individual. When the child begins his school, life is where you can see more in detail these difficulties, since that is where the processes take place taking into account that the oral language involves the faculty that must have the human being to learn new knowledge of the world that surrounds you. It is for this reason that the objective of this article is to address and explain the relationship of sensory integration with learning disorders more specifically the reading and writing code and its importance in the teaching-learning process, where a literary review of different types of scientific research in the international and national field, where it is evident that a deficit in sensory processing influences a large percentage of negative way in the cognitive processes that allow a meaningful learning to be successfully carried out.
\end{abstract}

Keywords: learning disorders; lectographic code; sensory integration.

\section{Resumo}

A integração sensorial é a habilidade do cérebro de integrar e processar de maneira apropriada os estímulos recebidos do ambiente e gerar as respostas apropriadas que são necessárias em um determinado momento; É por isso que, para que, quando disse que a integração sensorial é inadequada, ineficiente ou irregular, pode afetar significativamente a vida de um indivíduo. Quando a criança começa a vida escolar é o lugar onde você pode mostrar mais detalhadamente estas dificuldades, pois é onde os processos lectográficos ocorrem uma vez que a linguagem oral envolve corpo docente deve possuir o ser humano a aprender novos conhecimentos do mundo rodeia. É por esta razão que o objetivo deste artigo é abordar e explicar a relação de integração sensorial com dificuldades de aprendizagem, mais específicamente o código lectoescrito e sua importância no processo de ensino e aprendizagem, onde uma revisão da literatura de diferente aconteceu tipos de pesquisa científica em nível nacional e internacional, onde é evidente que um déficit no processamento sensorial uma grande influências percentuais negativamente sobre os processos cognitivos que permitem a conclusão bem sucedida da aprendizagem significativa.

Palavras-chave: código lectográfico, integração sensorial, distúrbios de aprendizagem. 


\section{Introducción}

La educación es un proceso fundamental en la vida del ser humano. Durante los primeros años de vida el niño inicia su actividad escolar, lo que permite el desarrollo de aspectos biológicos, sociales y afectivos. También de la mano de esta actividad tiene lugar el desarrollo de su personalidad, que se produce a través de las diversas experiencias que va viviendo, a lo largo del proceso madurativo, en su habilidad motriz, intelectual y emocional. Todo esto explica por qué recibir una educación adecuada y ajustada a las necesidades individuales es tan importante para la vida de cualquier persona. Los trastornos del aprendizaje actualmente son una preocupación tanto para el padre de familia, quien no comprende qué ocurre con su hijo, como para el docente, quien muchas veces tampoco sabe qué ocurre con el estudiante ni cómo ayudarlo de manera adecuada en su proceso de aprendizaje.

Esto pone de manifiesto la necesidad, por un lado, de que las instituciones educativas y los padres de familia unan esfuerzos y participen de manera activa en este proceso. Por otro lado, también es muy importante no dejar de lado cómo se siente el niño al ver que no alcanza dicho aprendizaje al ritmo de sus compañeros, lo cual puede afectarlo emocional y socialmente. En este artículo se expondrá la importancia de la integración sensorial para el buen procesamiento de la información que el cerebro recibe del entorno a través de los sentidos: esto es fundamental para la adquisición de los códigos lectoescritos, que se desarrollan a través de unas estructuras en el sistema nervioso central (SNC) cuya producción va aumentando a medida que el niño va recibiendo dichos estímulos.

Así mismo se revisará la participación de estructuras del cerebro como la corteza premotora, que se encarga de transmitir la información al área motora primaria y de responder a estímulos visuales y somato sensoriales, preparando cuerpo, cabeza y ojos para la coordinación de las tareas, y usando el tono muscular, el equilibrio y otras funciones esenciales para el aprendizaje de tipo motor. Dentro de los trastornos del aprendizaje se citarán los relacionados con el desarrollo del lenguaje. Estos se pueden dar como consecuencia de una estimulación inadecuada o pobre desde los primeros años de vida, dificultando que el niño pueda expresarse y hablar de forma correcta. En estos casos los niños requieren un entrenamiento permanente, y que los adultos de su entorno sepan escucharlos, pronunciando las palabras de forma correcta e interactuando con ellos a través de la lectura de cuentos, canciones y frases de afecto.

Las dificultades de aprendizaje y los trastornos del desarrollo muchas veces se confunden. La dificultad de aprendizaje hace referencia a que el niño a nivel académico se encuentra por debajo de la media respecto a sus compañeros y la edad que tiene, y el trastorno se refiere a que el niño no supera la dificultad a pesar de tener el apoyo que requiere. 
Dentro de los trastornos de lenguaje se mencionarán los relacionados con los trastornos primarios, que hacen referencia a la dislalia funcional, retraso del habla, retraso del lenguaje, disfasia, disglosias, disartrias y por último las afasias (Gil, 2007).

\section{Antecedentes}

Los niños son seres humanos únicos e irrepetibles, en proceso de crecimiento y desarrollo, y van avanzando por unas etapas que, una vez superadas, le permitirán un mayor aprendizaje y adaptación al mundo que los rodea. Tanto el desarrollo embrionario como el resto del ciclo vital se van modificando a través de conexiones o redes neuronales, que van creando el substrato neurofisiológico necesario para el desarrollo de las funciones cognitivas y del comportamiento. Las sucesivas y complejas transformaciones que van ocurriendo en el sistema nervioso se inician en la etapa prenatal con la formación de unas estructuras fundamentales, genéticamente determinadas (procesos independientes de la actividad), y se continúan en la vida extrauterina con la intervención del ambiente, que será el responsable del refinamiento de los circuitos neuronales (procesos dependientes de actividad), hasta conseguir su funcionalidad completa.

Gracias a esta plasticidad neuronal que se entiende como la propiedad de las neuronas para establecer algún tipo de comunicación — se logra modular la percepción de los estímulos con el medio, lo que se hace tanto a partir de factores intrínsecos como extrínsecos (en los que sobresalen los factores ambientales). Estos, en condiciones normales, modulan el desarrollo anatómico y funcional del sistema nervioso. En definitiva, el cerebro se desarrolla a partir de una compleja interacción entre el capital genético y las experiencias que se brindan a los niños, y por eso la calidad de los estímulos es fundamental para el desarrollo de las capacidades presentes y futuras de los infantes.

Durante dicho desarrollo algunos niños presentan un desenvolvimiento inadecuado, lo que significa que el cerebro no está funcionando de manera eficiente. Esta alteración afecta la integración de los sistemas sensoriales, ocasionando trastornos de aprendizaje en el proceso académico y sobre todo en el proceso de adquisición de los códigos para la lectoescritura. Bellefeuille (2006) expone una novedosa teoría, denominada "Teoría de la Integración Sensorial": él explica que la integración sensorial (Is) es la base para el desarrollo cognitivo-perceptual y sensorio-motor; la integración, además, es la que exige a cada individuo la interpretación adecuada de la información sensorial que llega al sistema nervioso - tanto a través de su propio cuerpo como del contexto-, necesaria para la planificación de las acciones adaptativas exigidas por el ambiente.

De igual forma Ramírez (2016), quien cita a Dunn, establece que para generar unas respuestas adaptativas es necesario 
un adecuado procesamiento sensorial: él propone un modelo que relaciona las respuestas del comportamiento y/o adaptativas con las formas utilizadas por el sistema nervioso para registrar, percibir, organizar e integrar la información sensorial. En esta línea, las disfunciones en el procesamiento sensorial — como la generación de híper o hipo respuestas a ciertos tipos de estímulos- se relacionarían con dificultades en el aprendizaje, que viene a ser un producto de alto nivel para la integración sensorial. Estas dificultades se evidencian en problemas con la lectura, la escritura, el cálculo, el razonamiento lógico, la comprensión, la memorización, el déficit de atención, la inquietud motora, las respuestas emocionales inapropiadas, los problemas en la secuenciación, la planeación y anticipación, entre otros.

Daniela Ramírez Bayona (2016) realiza un estudio en el que participan niños escolares con epilepsia entre los 7 y 10 años, y encuentra en el grupo de "Epilepsia no controlada versus controles y epilepsia controlada" unas correlaciones significativas entre alteraciones de modulación sensorial (pobre registro, búsqueda sensorial y evitador sensorial) y dificultades de aprendizaje (orientación, percepción espacial, praxia y construcción viso-motriz). Los hallazgos de esta investigación coinciden con las escasas investigaciones previas: también hablan sobre una mayor presencia de alteraciones en la modulación sensorial en niños con epilepsia frente a niños sanos.
Del mismo modo, Caicedo (2017) realiza un estudio con niños de 7 a 9 años, identificando a través de la evaluación de procesamiento sensorial (eps) que el $32 \%$ de los alumnos presenta dificultades de is; dentro de los datos que observó destaca que un 28 \% presenta dificultades en el procesamiento visual y un $35 \%$ dificultad en errores específicos. Con respecto a la lectoescritura se logró identificar, a través de la evaluación neuropsicológica infantil (eni), que un $72 \%$ de los estudiantes presenta dificultad en rendimiento académico, a lo que se suman las dificultades de motricidad fina y gruesa, lenguaje expresivo y comprensivo. Además, un 33 \% tiene dificultad para la comprensión lectora, un 48 \% presenta dificultad para la velocidad lectora, un $30 \%$ presenta dificultad para la precisión escrita, un $38 \%$ tiene dificultad para la composición narrativa y un $43 \%$ presenta dificultad para la velocidad de escritura.

Erazo (2016), a través de su estudio sobre la identificación y descripción de las relaciones entre integración sensorial, atención y conducta, identifica que el $64 \%$ de la muestra tiene déficit de integración sensorial; el 98\% problemas de motricidad fina y el 94 \% en cálculo; además, un $22 \%$ presenta problemas de atención y un porcentaje entre el $6 \%$ y el $45 \%$ manifiesta dificultades de conducta y relaciones sociales. Existen interacciones significativas entre las variables medidas, y como conclusión principal Erazo establece que los estudiantes de la muestra presentan dificultades de comportamiento adaptable a las necesidades 
del aula, aprendizaje y comportamiento, lo que tiene relación con déficits neuropsicológicos y de madurez.

Por otro lado, Cancino y Ramos (2007) realizaron un estudio en Chile para describir el procesamiento sensorial y el rendimiento escolar en niños entre 7 y 9 años. Ellos buscaban definir la probabilidad de disfunción del procesamiento sensorial con respecto al rendimiento escolar en dicha población, y para ello llevaron a cabo un estudio no experimental de tipo transeccional-descriptivo. Se estudió una muestra de 63 niños de segundo año de educación general básica mediante la realización de un cuestionario sobre los antecedentes generales del niño, y en el cual se incluyó el promedio de notas del primer semestre del año en curso, y la aplicación de la evaluación de procesamiento sensorial de Parham y colaboradores. La evaluación de procesamiento sensorial de los autores Parham y Ecker es un test que permite obtener información acerca del tipo de respuestas que los niños presentan en relación a diferentes eventos sensoriales. Es decir que la manera en que un niño procesa y responde a la información sensorial del entorno y de su propio cuerpo debe formar parte de toda la evaluación del perfil de un niño en los diferentes contextos. Se encontró que los niños con bajo rendimiento presentan una alta probabilidad de disfunción del procesamiento sensorial en comparación con el grupo de alto rendimiento, que presentó un menor porcentaje. Por último, se realizó un análisis cualitativo de las variables que condujo al rechazo de la hipótesis nula de la prueba estadística, estableciéndose la existencia de una asociación entre las variables analizadas.

Actualmente no se encuentran estudios concluyentes sobre el tema de la desintegración sensorial del código lectoescrito, por ello se considera realizar esta revisión. El fin es establecer la relación existente entre la integración sensorial y los trastornos en el aprendizaje del código lecto-gráfico en un estudio a niños escolarizados de 6 a 9 años. El resultado brindaría respuestas sobre la participación de las estructuras cerebrales en el proceso de la integración sensorial, y permitiría tanto establecer la relación entre la integración sensorial y los procesos psicológicos superiores que intervienen en el aprendizaje, como determinar las causas de los problemas en lectoescritura y su influencia en el desarrollo de los niños en etapa escolar; por último, también se establecería la importancia de la integración sensorial y su relación con el proceso de aprendizaje de la lectoescritura.

Una disfunción en la integración sensorial dificulta al ser humano su adaptación al ambiente de una manera adecuada, lo que se debe a un desarrollo incorrecto en el cerebro de aquellos procesos que integran las sensaciones. Esto explica sus consecuencias: retrasos en el desarrollo psicomotor, déficit en el rendimiento sensorio-motor, disfunción en la percepción, limitaciones en el funcionamiento mental y las capacidades adaptativas, y dificultades en el aprendizaje académico. Y dichas consecuencias conllevan, a su vez, a la 
aparición de limitaciones en actividades de la vida cotidiana como el juego, el ocio, la educación y la participación social de los niños, lo que afecta su desempeño en las diversas tareas. La intervención sensorial en los problemas de aprendizaje en la infancia fueron algunos de los primeros resultados que abordó la is, principalmente en las áreas de matemáticas y lectura (Troncoso, 2014). Troncoso (2014) plantea que la intervención sensorial en los problemas de aprendizaje en la infancia fueron algunos de los primeros resultados que aborda la integración sensorial principalmente en las áreas de matemáticas y lectura y que uno de los objetivos de Jean Ayres quien es la precursora de la teoría de integración sensorial, era describir, explicar y predecir la relación entre el funcionamiento neurológico, el comportamiento sensorio-motor y el aprendizaje académico.

Ayres (2005) establece que el cerebro tiene la capacidad para integrar y procesar información de manera innata a través del control del input sensorial de sistemas como el vestibular, propioceptivo y táctil; por tanto, este determina la habilidad que permite que el niño desarrolle interacciones eficaces con los diferentes actores de su ambiente, y genere respuestas adaptativas en relación con el entorno en que se desenvuelve. El cerebro emprende procesos de selección, organización y asociación de la información sensorial, mediante patrones flexibles y continuamente cambiantes que van dando paso a sistemas de funcionamiento cada vez más complejos: si estos presen- tan irregularidades o disfuncionalidades pueden afectar de manera significativa los procesos de aprendizaje y las relaciones interpersonales de un niño.

En este sentido, Smith y Kosslyn (2008) consideran que cada hemisferio cerebral está conformado por cuatro lóbulos: el occipital recibe e interpreta los estímulos visuales; el temporal almacena los datos y experiencias en la memoria, atribuye significados y permite comprender las situaciones; el parietal facilita la representación espacial, la integración de información táctil y kinestésica del lado contralateral del cuerpo, y determina los procesos atencionales y de razonamiento matemático; y el frontal se encarga de organizar las secuencias de conducta, controlar los movimientos y buscar información específica almacenada, siendo además la base para la toma de decisiones, pues está involucrado en la programación, regulación y verificación de la actividad mental.

Ardila y Ostrosky (1991) se refieren a la organización de las estructuras cerebrales, resaltando que, a pesar de la importancia de la corteza cerebral para el pensamiento abstracto, todo el sistema nervioso central contribuye a su formación mediante la existencia de tres tipos de zonas corticales: primarias (áreas específicas donde se proyecta información de los sistemas sensoriales visual, auditivo y somestésico), secundarias (áreas de conexiones neuronales transcorticales, que analizan e integran los mensajes en percepciones y experiencias reconocibles y provistas de significado) y terciarias (áreas de 
cruce de información de distintos sistemas sensoriales que contribuyen en la integración multimodal, y que están ubicadas en la región frontal y en la parieto-temporo-occipital).

Lo anterior pone de manifiesto cómo la integración sensorial está ligada a todos los procesos corticales, lo que se refleja en acciones como: el registro o capacidad para percibir el estímulo a través de sensorios; la modulación-regulación o habilidad para organizar la intensidad y naturaleza de las respuestas gracias a mecanismos de excitación e inhibición cortical; la discriminación o interpretación de la información que determina su relevancia, características y cualidades específicas; y la integración o asociación de estímulos significativos, que interpreta las demandas del ambiente y posibilita a su vez, una respuesta adecuada del organismo (Erazo, 2016).

Moya y Matesanz (2010) plantean que el concepto de integración sensorial se define como la habilidad para organizar la información sensorial para su uso; posteriormente fue revalorada y se definió como el proceso neurológico que organiza la sensación de nuestro propio cuerpo y del ambiente, y que además hace posible que el cuerpo la use efectivamente dentro de sí mismo. Es decir, el buen funcionamiento de nuestro cerebro al procesar esta información nos permite producir continuamente 40 respuestas adaptadas a nuestro entorno y a las cosas que en él tienen lugar, lo que constituye la base del aprendizaje académico y del comportamiento social.
En otras palabras, el procesamiento realizado por el sistema nervioso hace que la información se reciba desde las diferentes modalidades sensoriales, por lo que el cerebro debe seleccionar, aumentar, inhibir, comparar y asociar toda la información sensorial en un patrón flexible, constante y cambiante, integrándolas todas. Por lo general, cuando se hace referencia a los sentidos se piensa en el tacto, olfato, el gusto y la audición, pero se desconoce el vestibular y el propioceptivo. Antes de aprender a leer, escribir y calcular, se tiene que dar un significado a lo que se ve o escucha, se debe ser capaz de planificar los movimientos y organizar el comportamiento. Esta capacidad depende de la eficacia con la que el sistema nervioso organiza los mensajes que son transmitidos. La integración sensorial es la capacidad del niño para sentir, comprender y organizar la información sensorial proveniente de su cuerpo y su entorno.

Por otro lado, Vidal (2014), con base en los planteamientos de Fodor, García y Morán (1997), concluye que estas informaciones se transmiten tanto por los sistemas sensoriales — como la visión, el tacto, el olor, el gusto, la audicióncomo por los sistemas vestibulares y propioceptivos. La capacidad de la integración sensorial depende de la eficacia con la que el sistema nervioso organiza los mensajes que recibe. Una adecuada integración organiza las informaciones sensoriales necesarias para que el niño se desarrolle armoniosamente, o sea que la integración neurosensorial es funda- 
mental para el buen funcionamiento comunicativo. La integración sensorial se desarrolla sin esfuerzo en el curso de las actividades ordinarias de los niños, pero en ocasiones el cerebro presenta dificultades para procesar la información entrante y puede existir un inadecuado registro de los estímulos sensoriales, cualquiera que estos sean.

La integración sensorial inicia en la matriz cuando el feto siente los movimientos del cuerpo de la madre; una enorme cantidad de integración sensorial debe ocurrir y desarrollarse para que el niño pueda gatear y ponerse de pie, y esto sucede durante el primer año de vida. Los juegos de la infancia conducen a mucha integración sensorial en la medida en que el niño va organizando sus sensaciones de cuerpo y gravedad junto con el sentido de la visión y el oído. Posteriormente, en la etapa escolar, durante la lectura se requiere de una compleja integración de sensaciones de los ojos, músculos, cuello y de un órgano sensorial muy especial localizado dentro de los oídos. Es importante mencionar que los genes de la especie humana nos proporcionan una plataforma para la capacidad de integración sensorial, fruto de las abundantes interacciones con el mundo y las progresivas adaptaciones del cuerpo y el cerebro humanos a los retos físicos de la infancia. El mayor desarrollo de integración sensorial ocurre durante una respuesta adaptativa, que es una experiencia sensorial provista de un propósito y una meta; al mismo tiempo la formación de una respuesta adaptativa ayuda a la propia organización y al desarrollo del cerebro. El juego produce respuestas adaptativas que dan lugar a la integración sensorial: el niño que aprende a organizar su juego tiene más posibilidades de organizar su trabajo en la escuela y volverse un adulto organizado (Ayres, 2005).

Respecto a los trastornos específicos del desarrollo del aprendizaje escolar, Magaña y Ruiz-Lazaro (2018) establecen que son trastornos producto de un deterioro de las formas normales del aprendizaje desde las primeras etapas del desarrollo. El deterioro no solo se produce por falta de oportunidades de aprendizaje o traumas o enfermedades cerebrales adquiridas, sino que también surge por alteraciones —en gran medida secundarias a algún tipo de disfunción biológica- de los procesos cognoscitivos. Su etiología no es conocida, pero se acepta el predominio de los factores biológicos en interacción con otros como las oportunidades para aprender y la calidad de la enseñanza. Si bien la escuela es un factor que se debe considerar, los trastornos no pueden reducirse puramente a errores pedagógicos. La forma en que estos trastornos se presentan varía con la edad; es habitual que un trastorno del habla y el lenguaje en edad preescolar pierda intensidad y se prolongue en el tiempo en forma de un retraso de la lectura, que en la adolescencia se aminora y da lugar en la edad adulta a un trastorno de la ortografía. Es necesario para el diagnóstico que los trastornos se manifiesten de alguna manera durante los primeros años de escolaridad. 
La lectura, la escritura y la aritmética resultan ser estrategias complejas incluso para la mayoría de los niños que aprenden a leer, a escribir y a calcular (sumar, restar, multiplicar y dividir) sin dificultad; contando con un grado

\section{Tabla 1. Clasificación según su etiología}

\begin{tabular}{|c|c|}
\hline \multirow{7}{*}{$\begin{array}{l}\text { Dificultades } \\
\text { de origen } \\
\text { primario }\end{array}$} & $\begin{array}{l}\text { Déficit intelectual en el límite de la normalidad, cociente intelectual o CI muy alto, } \\
\text { bajo o muy bajo. }\end{array}$ \\
\hline & Problemas neurológicos. \\
\hline & Enfermedades crónicas o incapacidad física. \\
\hline & Enfermedades crónicas o incapacidad física. \\
\hline & Déficit sensorial: auditivo y/o visual. \\
\hline & $\begin{array}{l}\text { Enfermedades carenciales: malnutrición, ferropenia, alteraciones tiroideas (apatía, } \\
\text { somnolencia). }\end{array}$ \\
\hline & $\begin{array}{l}\text { Rinitis crónica, que suele ocasionar problemas para oír, aprender a hablar y pronunciar } \\
\text { correctamente. }\end{array}$ \\
\hline \multirow{8}{*}{$\begin{array}{l}\text { Dificultades } \\
\text { específicas. }\end{array}$} & En aptitudes escolares: \\
\hline & $\begin{array}{l}\text { Para el aprendizaje del cálculo: discalculia. Son alumnos que tienen dificultad para sumar } \\
\text { y restar: para efectuar operaciones de cálculo. Confunden los números, los invierten o } \\
\text { los escriben al revés. }\end{array}$ \\
\hline & $\begin{array}{l}\text { Para el aprendizaje de la escritura: digrafía. Nivel de escritura inferior al que les corresponde, } \\
\text { omiten letras o juntan palabras. Hay distorsión en el orden y posición de las palabras. }\end{array}$ \\
\hline & $\begin{array}{l}\text { Para el aprendizaje y desarrollo de la lectura fluida y comprensiva: dislexia. Es una } \\
\text { dificultad para aprender a leer y a escribir. }\end{array}$ \\
\hline & $\begin{array}{l}\text { Disortografía. Imposibilidad de aplicar las reglas ortográficas. Secuela de la dislexia aun } \\
\text { después de ser superada. }\end{array}$ \\
\hline & $\begin{array}{l}\text { Disfasia. Retraso en la aparición del lenguaje oral y escrito, asociado a problemas perceptivos. } \\
\text { Desarrollo del lenguaje receptivo. }\end{array}$ \\
\hline & $\begin{array}{l}\text { Motoras: desarrollo de la coordinación motora fina y/o gruesa presentando problemas de } \\
\text { lateralidad o falta de coordinación visomotora, con dificultad en distinguir dónde está la } \\
\text { derecha y dónde la izquierda, en manos, pies, ojos, etc.; deficiencia en la organización } \\
\text { espacial (no sabe dibujar con perspectiva y tiene dificultades para localizar objetos) o } \\
\text { en la temporal por un retraso psicomotriz. }\end{array}$ \\
\hline & $\begin{array}{l}\text { Aunque no es un trastorno específico del aprendizaje nos referiremos someramente al } \\
\text { TDA (trastorno por déficit de atención, con o sin hiperactividad). El TDAH adquiere gran } \\
\text { importancia por el elevado porcentaje que representa en las estadísticas como causa de } \\
\text { fracaso escolar. La tríada característica es: actividad motora excesiva; déficit de atención; } \\
\text { impulsividad. Son síntomas asociados, entre otros: trastornos de conducta; dificultades de } \\
\text { aprendizaje; problemas de relación social; bajo nivel de autoestima; alteraciones emocionales. }\end{array}$ \\
\hline $\begin{array}{l}\text { Dificultades } \\
\text { derivadas } \\
\text { del entorno } \\
\text { socio- } \\
\text { familiar y } \\
\text { cultural. }\end{array}$ & $\begin{array}{l}\text { Procesos educativos no satisfactorios. } \\
\text { Lenguaje en el hogar. } \\
\text { Salud. } \\
\text { Atmósfera de aprendizaje en casa. } \\
\text { Problemas familiares. } \\
\text { Cambios de escuela. } \\
\text { Calidad de la escuela. }\end{array}$ \\
\hline
\end{tabular}

Fuente: Magaña y Ruiz-Lazaro (2018). 
de maduración determinada y con un ambiente pedagógico favorable, los niños son capaces de acceder sin grandes problemas al dominio de la lectoescritura y el cálculo. Pero, tanto la lectura como la escritura o la aritmética ("las mates") pueden llegar a convertirse en un problema para niños normales en otros aspectos de su desarrollo evolutivo.

La denominación más amplia de "problemas escolares" tiene tantos registros como la vida de cada persona. Incluye la capacidad personal y de adaptación a los aprendizajes de cada alumno, con sus rasgos concretos y únicos de personalidad, y con las características fisiológicas y psicológicas específicas del desarrollo de cada etapa de la vida en referencia al mundo escolar - hasta haber completado las etapas de enseñanza el estudiante pasará un tercio de cada día en la escuela-. En el manual diagnóstico y estadístico de los trastornos mentales (DSMV) desaparece la categoría de los trastornos de inicio en la infancia, la niñez y la adolescencia, y aparecen los trastornos del neurodesarrollo, donde se incluye, entre otros, el trastorno específico del aprendizaje. Esto explica la importancia de una detección a tiempo, así como una actuación perentoria sobre todos estos problemas, de manera que se pueda lograr una integración social y escolar adecuada, que es la única forma de prevenir las dificultades escolares (que pueden, a su vez, dificultar también el futuro profesional). Estas dificultades se deben abordar directamente dentro de la escuela con programas individua- lizados para cada dificultad específica y siempre en colaboración con los profesionales específicos en cada área.

Dentro de los trastornos del lenguaje escrito están el retraso lector, la disgrafía, la disortografía y la dislexia. El lenguaje escrito es un sistema de comunicación representado a través de signos gráficos que permite trasmitir información, ideas y sentimientos; requiere del desarrollo adecuado de una serie de variables mentales, cognitivas, lingüistas, metalingüísticas y sensoemocionales. La disgrafía es una alteración en la calidad del trazo gráfico que no implica la existencia de un retraso neurológico o intelectual que lo justifique; igualmente, sin embargo, puede retrasar el avance escolar. Se asocia a un trastorno psicomotor (dispráxico), así como a la zurdería y los trastornos de lateralidad. Los grafismos son confusos: abiertos, retocados, tamaño grande, pequeño o muy pequeño, tembloroso, fluctuante y, asociado a esto, se puede percibir un manejo irregular del espacio entre renglones y márgenes. No todos los niños con dificultad para componer textos escritos poseen este tipo de trastorno, pues una instrucción deficiente, la falta de experiencia para manipular las estructuras del lenguaje o un problema cognoscitivo o emocional generalizado pueden afectar también la expresión escrita (Ardila, Roselli y Matute, 2005).

Existen distintos tipos de disgrafía: 
Tabla 2. Tipos de disgrafia

\begin{tabular}{|c|c|}
\hline $\begin{array}{l}\text { TIPO DE } \\
\text { DISGRAFÍA }\end{array}$ & ORIGEN \\
\hline $\begin{array}{l}\text { Disgrafía } \\
\text { postural }\end{array}$ & - Se origina por una postura inadecuada al escribir. \\
\hline $\begin{array}{l}\text { Disgrafía de } \\
\text { prensión }\end{array}$ & 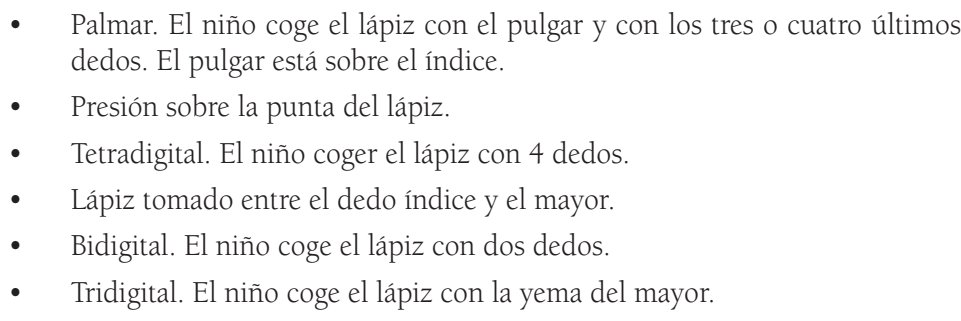 \\
\hline $\begin{array}{l}\text { Disgrafía } \\
\text { de presión }\end{array}$ & $\begin{array}{l}\text { - } \quad \text { Trazos débiles. Alas de mosca. } \\
\text { - } \quad \text { Excesiva presión en el trazo al escribir. Letras aplastofolio. } \\
\text { - } \quad \text { Letra parkinsoniana. Pequeña, temblorosa y rígida. }\end{array}$ \\
\hline $\begin{array}{l}\text { Disgrafía } \\
\text { de direccio- } \\
\text { nalidad }\end{array}$ & $\begin{array}{l}\text { - } \quad \text { Descendente. } \\
\text { - } \quad \text { Ascendente. } \\
\text { - Serpenteante. }\end{array}$ \\
\hline $\begin{array}{l}\text { Disgrafía } \\
\text { de giro }\end{array}$ & $\begin{array}{l}\text { - Las letras que necesitan trazos circulares en su ejecución se realizan con giros } \\
\text { invertidos, es decir, en el sentido de las agujas del reloj. } \\
\text { - Dificultades en el trazo y su enlace con la siguiente. }\end{array}$ \\
\hline $\begin{array}{l}\text { Disgrafía } \\
\text { de enlace }\end{array}$ & $\begin{array}{l}\text { - } \quad \text { Letras que no se enlazan en cursiva. } \\
\text { - } \quad \text { Simbiótico. Letras pegadas entre sí. } \\
\text { - Elástico. Letras separadas y unidas forzosamente con líneas que parecen } \\
\text { sobreagregadas. }\end{array}$ \\
\hline $\begin{array}{l}\text { Disgrafía } \\
\text { figural }\end{array}$ & - Mutilaciones y distorsiones de letras. \\
\hline $\begin{array}{l}\text { Disgrafía } \\
\text { posicional }\end{array}$ & - Verticalidad, caída hacia atrás, letras en espejo, confusión de letras simétricas. \\
\hline $\begin{array}{l}\text { Disgrafía } \\
\text { relaciona- } \\
\text { da con el } \\
\text { tamaño }\end{array}$ & $\begin{array}{l}\text { - } \quad \text { Macrografía. } \\
\text { - } \quad \text { Micrografía. }\end{array}$ \\
\hline $\begin{array}{l}\text { Disgrafía } \\
\text { espacial }\end{array}$ & $\begin{array}{l}\text { - Interlineado irregular. } \\
\text { - } \quad \text { Texto orillado a la izquierda. }\end{array}$ \\
\hline
\end{tabular}

Fuente: Ardila, Roselli y Matute (2005). 
La disortografía es un trastorno específico de la escritura que condiciona y dificulta la escritura del niño: se trascribe el código escrito de forma inexacta en relación con la norma ortográfica porque no se asocian sonido y grafía. Este trastorno se percibe de manera recurrente y exacerbada - las palabras o el texto pueden llegar a ser inteligibles-. Las causas de la disortografía pueden ser de tipo intelectual, lingüístico o pedagógico. Giner (2008) cita a Luria y Tsvetkova, quienes distinguen siete tipos de disortografía, cuyos aspectos fundamentales se describen en la tabla 3.

\section{Tabla 3. Tipos de disortografía}

\begin{tabular}{|l|l|}
\hline $\begin{array}{l}\text { TIPOS DE } \\
\text { DISORTOGRAFÍA }\end{array}$ & DESCRIPCIÓN \\
\hline Disortografía temporal & $\begin{array}{l}\text { Se encuentra relacionada con la percepción del tiempo y del ritmo, } \\
\text { presentando dificultades en la percepción de los aspectos fonémicos de } \\
\text { la cadena hablada y su correspondiente transcripción escrita, así como } \\
\text { la reparación y unión de sus elementos. }\end{array}$ \\
\hline $\begin{array}{l}\text { Disortografía } \\
\text { perceptivo-cinestésica }\end{array}$ & $\begin{array}{l}\text { Está relacionada con dificultades relativas a la articulación de los fonemas } \\
\text { y a la discriminación auditiva de éstos. } \\
\text { Se presentan frecuentemente los errores de sustitución de letras "r" por } \\
\text { "l", sustituciones que suelen dar asimismo en el habla. }\end{array}$ \\
\hline $\begin{array}{l}\text { Disortografía } \\
\text { disortocinética }\end{array}$ & $\begin{array}{l}\text { Se encuentra alterada la secuenciación fonemática del discurso a nivel } \\
\text { de ordenación y secuenciación de los elementos gráficos, provocando } \\
\text { errores de unión o fragmentación de palabras. }\end{array}$ \\
\hline visortografía & $\begin{array}{l}\text { Se relaciona con la percepción visual y de forma más específica con la } \\
\text { orientación espacial, incidiendo esto en la correcta percepción de de- } \\
\text { terminadas letras o grafemas, y pudiendo producir errores de rotación } \\
\text { de letras como las frecuentes rotaciones de "b" por "d" o de "p" por "q". } \\
\text { Se pueden presentar sustituciones de grafemas con una forma parecida } \\
\text { como "a" por "o" o "m" por "n". } \\
\text { También se encuentran los errores propios de las inversiones de letras } \\
\text { en la escritura de determinadas palabras. }\end{array}$ \\
\hline $\begin{array}{l}\text { Disortografía } \\
\text { semántica }\end{array}$ & $\begin{array}{l}\text { Se altera el análisis conceptual de las palabras, pudiéndose producir } \\
\text { uniones y fragmentaciones de palabras, así como el uso de señales } \\
\text { diacríticas o signos ortográficos. } \\
\text { Disortografía dinámica } \\
\text { expresión escrita a nivel de gramática: el orden de los elementos en la } \\
\text { elementos relevantes en la oración. }\end{array}$ \\
\hline $\begin{array}{l}\text { Disortografía cultural y número o la omisión de los } \\
\text { acentuación o uso de determinadas letras. }\end{array}$ \\
\hline Es lancapacidad para el aprendizaje de la normativa ortográfica, ya sea \\
\hline
\end{tabular}

Fuente: Luria y Tsvetkova, citados por Giner (2008). 
La dislexia es un problema específico del aprendizaje, que ha sido definida por la Federación Mundial de Neurología como un desorden que se manifiesta en dificultades para aprender a leer a pesar de una instrucción convencional, inteligencia adecuada y oportunidad sociocultural. Depende de las dificultades cognoscitivas fundamentales, que frecuentemente son de origen construccional. (Ardila, Roselli y Matute, 2005). Se dice que un niño es disléxico cuando presenta dificultades en el aprendizaje de la lectoescritura, independientemente de que tenga un desarrollo inelectual adecuado para este proceso. Se considera dislexia cuando se presenta una demora en el reconocimiento del fonema y la transformación del morfema. Además se pueden presentar: agregados, cuando se aumentan letras o combinaciones; disociaciones, separaciones inadecuadas; problemas en la pronunciación de palabras familiares; problemas en la identificación de la primera letra con cambio de la palabra; deletreo alterado de una palabra después de escucharla; dificultad para hacer rimas; $y$ dificultad al recitar el alfabeto. Se pueden considerar dos tipos de dislexia.

\section{Tabla 4. Tipos de dislexia}

\begin{tabular}{|c|c|c|}
\hline TIPO DE DISLEXIA & CLASES & \\
\hline \multirow[t]{2}{*}{$\begin{array}{l}\text { Las dislexias y } \\
\text { disgrafías visomotoras } \\
\text { son dificultades } \\
\text { en la percepción } \\
\text { visoespacial a nivel } \\
\text { gnosias y praxias. }\end{array}$} & $\begin{array}{l}\text { Disguestálticas. } \\
\text { Las fallas gnósicas } \\
\text { se presentan en el } \\
\text { procesamiento visual. }\end{array}$ & $\begin{array}{l}\text { Dificultad en la orientación espacial. } \\
\text { Fallas en el esquema corporal. } \\
\text { Alteraciones de lateralidad. } \\
\text { Rotaciones. } \\
\text { Pérdida en la ubicación de la lectura del texto. } \\
\text { Fallas en la discriminación figura fondo. } \\
\text { Inversiones. } \\
\text { Omisiones. } \\
\text { Unir letras, sílabas y palabras. } \\
\text { Distorsiones. } \\
\text { Deformaciones. } \\
\text { Dificultad para asociar fonema y grafema. } \\
\text { Falla en la copia. } \\
\text { Disnodia de integración. } \\
\text { No identifica el error. }\end{array}$ \\
\hline & $\begin{array}{l}\text { Grafomotoras. } \\
\text { Alteraciones en } \\
\text { las praxias. }\end{array}$ & $\begin{array}{l}\text { Dificultad al escribir. } \\
\text { Dificultad de ordenamiento secuencial. } \\
\text { Alteraciones en la motricidad fina. } \\
\text { Dispraxias ideomotoras. }\end{array}$ \\
\hline
\end{tabular}

Fuente: Luria y Tsvetkova, citado por Giner (2008). 
La desintegración sensorial incide en el código lectoescrito en niños de 6 a 9 años; según lo que expresa Ayres (2005) el reconocimiento sensorial que realiza el sujeto en sus primeras experiencias aporta al fortalecimiento de la construcción de habilidades motoras y cognitivas, brindando las herramientas necesarias para potencializar el proceso de aprendizaje. Así mismo Serna, Torres y Torres (2017) citan a De Burgos y Bonorandi, quienes refuerzan la idea propuesta por Ayres (2005), y exponen que los primeros siete años conforman el periodo más importante en el proceso de integración sensorial; para esta consideración se tienen en cuenta dos factores fundamentales: el primero es la capacidad que presenta el cerebro para registrar sensaciones y el segundo es la capacidad aumentada con la que se cuenta para reorganizar la información obtenida. Así, se trata de un periodo clave porque en éste las funciones sensorio-motoras se consolidan y se convierten en la base para capacidades intelectuales avanzadas (Serna, Torres y Torres, 2017).

Durante el proceso de aprendizaje es probable que los niños presenten alteraciones desde su sistema subcortical, encargado del procesamiento de estímulos sensoriales y sus áreas de asociación -en las cuales dichos estímulos se concentran, con el fin de generar respuestas adaptativas-. Dichas debilidades se evidencian frecuentemente en la edad preescolar y escolar, momento en que la independencia en la ejecución de tareas aumenta. La causa de la alteración reside en la incapaci- dad del sistema nervioso central para interpretar y organizar adecuadamente la información captada por los diversos órganos sensoriales del cuerpo; en esta línea, tampoco puede analizar y utilizar dicha información adecuadamente para entrar en contacto con el ambiente, ni responder eficazmente a los múltiples estímulos del entorno (Beaudry, 2006).

Por lo anterior, el niño que presenta estas dificultades en la is encontrará dificultades en la interrelación con sus compañeros, docentes y familia, pues no puede codificar adecuadamente los estímulos provenientes del ambiente. Además, se presenta un déficit en la eficiencia de sus acciones y en la identificación de los mensajes y acciones de otros, lo que inhibe las respuestas adaptativas de sus sensaciones y por ende de su propio cuerpo: todo esto se manifiesta en las respuestas características del trastorno de procesamiento sensorial (Gamboa, Mónico y Triana, 2018). Estas dificultades son más evidentes cuando el niño ingresa a la escuela y se ve enfrentado a procesos cognitivos más complejos, como la adquisición del segundo código lingüístico (lectoescritura). Asimismo, se ven afectadas algunas de las funciones ejecutivas, como la atención, la memoria de trabajo o la inhibición, o sea las facultades necesarias para integrarse efectivamente en un entorno común con exigencias y regulaciones sociales. 
Tabla 5. Tipos de trastorno de procesamiento sensorial

\begin{tabular}{|c|c|c|}
\hline TRASTORNO & DEFINICIÓN & DIVISIÓN \\
\hline \multirow{3}{*}{$\begin{array}{l}\text { Trastorno de } \\
\text { modulación } \\
\text { sensorial }\end{array}$} & \multirow{3}{*}{$\begin{array}{l}\text { Se trata de una híper o } \\
\text { hiporesponsividad a sensaciones } \\
\text { habituales del cuerpo o del } \\
\text { entorno, incluyendo sensaciones } \\
\text { de tacto (tocar o ser tocado) y } \\
\text { de movimiento (mover o ser } \\
\text { movido). Son consecuencia de } \\
\text { la incapacidad del niño para } \\
\text { situarse en un umbral sensorial } \\
\text { que le permita interactuar } \\
\text { adecuadamente. Estas respuestas } \\
\text { pueden fluctuar entre hipo e híper. }\end{array}$} & Buscador de sensaciones. \\
\hline & & Hipo-reactividad sensorial. \\
\hline & & $\begin{array}{l}\text { Hiperreactividad sensorial. Miedoso o } \\
\text { cauteloso y/o en negativista desafiante. }\end{array}$ \\
\hline $\begin{array}{l}\text { Trastorno de la } \\
\text { discriminación } \\
\text { sensorial }\end{array}$ & $\begin{array}{l}\text { Se trata de la mala interpretación } \\
\text { u organización de la información } \\
\text { proveniente de la propia persona } \\
\text { y de su entorno. Esto produce } \\
\text { una dificultad para integrar } \\
\text { la información (feedback) } \\
\text { y, por tanto, para elaborar } \\
\text { respuestas adaptativas. }\end{array}$ & $\begin{array}{l}\text { Se ve afectada la interpretación } \\
\text { de la calidad de los estímulos } \\
\text { sensoriales, e impacta en la } \\
\text { habilidad de identificar similitudes } \\
\text { y diferencias entre los mismos. }\end{array}$ \\
\hline \multirow[t]{2}{*}{$\begin{array}{l}\text { Desorden motor } \\
\text { de base sensorial }\end{array}$} & \multirow{2}{*}{$\begin{array}{l}\text { Fruto de un déficit en la discrimina- } \\
\text { ción sensorial. Esta discriminación } \\
\text { sensorial deficitaria lleva a la dispraxia } \\
\text { o dificultad para idear, planificar o } \\
\text { llevar a cabo un acto motor o serie } \\
\text { de actos motores nuevos. }\end{array}$} & Dispraxia. \\
\hline & & Desorden postural. \\
\hline
\end{tabular}

Fuente: Pérez (2012) y Moya (2018).

Serna, Torres y Torres (2017) citan a Di Tore, Aiello, Paloma, Macchi y Sibilio, quienes establecieron que la importancia de la retroalimentación visual-motora puede considerarse como la primera integración sensorial del desarrollo. Esta cuenta con una base puramente sensorial; además, el canal vestibular es relevante para la adquisición de habilidades visuales, que proviene de la integración de procesos sensorio-perceptivo-motrices que son (a través del módulo de la aten48 ción) el origen del aprendizaje general y propio. El aparato vestibular se encarga de regular la postura, el equilibrio, el tono muscular y la orientación espacial, promoviendo el reconocimiento de figura-fondo y cierre visual, fortaleciendo a su vez habilidades motoras básicas con las que un niño logra iniciar procesos escritos y tareas de cálculo.

En esta línea, se entiende que el niño aprende inicialmente a través del movimiento y se expresa de manera gestual y corporal, logrando comunicarse con el medio. Cuando el niño tiene dificultades desde pequeño en la integración y exploración de diferentes espacios se altera el contacto con el mundo que lo rodea. Además, el desconocimiento de esta desintegración por parte de docen- 
tes, profesionales y familia no permite la habilitación o rehabilitación de los estudiantes con este tipo de déficit, ni tampoco ofrece la infraestructura o la preparación docente necesarias para desarrollar habilidades viso-motoras por medio de aprendizajes significativos (Lázaro, 2008). Es decir, actualmente el desconocimiento de algunos docentes, profesionales y padres de familia referente a lo que abarca la integración sensorial y sobre cómo estimular las habilidades viso-motoras y las vestibulares, sumado a una pobre infraestructura en algunas instituciones educativas, no contribuyen a desarrollar dichas habilidades ni permiten una verdadera rehabilitación en niños con déficit sensorial. En la mayoría de las ocasiones la inmadurez motora y sensorial con la que los niños se enfrentan a tareas escolares se constituye como un factor de riesgo para un proceso educativo que inicia en edades muy tempranas (Serna, Torres y Torres, 2017).

Redondo y Guerra, citados por Serna, Torres y Torres (2017) establecieron que otra de las áreas escolares que suele verse afectada es la educación física, pues cuando existe un trastorno de procesamiento sensorial (TPS) se hace evidente en la torpeza de la ejecución desde el primer desempeño sensorio-motor, generando angustia y rechazo en el niño. El fortalecimiento del canal vestibular es fundamental para lograr un adecuado desarrollo motor; este proceso no se podría dar sin retroalimentación sensorial continua, incluyendo estimulación vestibular, visual y propioceptiva, logrando que a nivel límbico el cerebro organice dicha información y genere en el sujeto respuestas motoras organizadas, sincronizadas y direccionadas a la adquisición y mantenimiento de diferentes posturas funcionales en su desempeño.

Así mismo se presentan dificultades en las habilidades práxicas en el aprendizaje del código lectoescrito, entendiendo a las praxis como procesos cognitivo-motores que requieren ideación, planeación y ejecución. Por lo dicho hasta acá, los problemas en la percepción táctil y el planeamiento motor tendrán consecuencias en estas habilidades (Serna, Torres y Torres, 2017). Asimismo, las dificultades en la integración perceptivo-motora afectarán aprendizajes de tipo no verbal. Los estudios han mostrado que dichas dificultades corresponden a una disfunción en el hemisferio derecho y generan un retraso en la adquisición de habilidades fundamentales para el desempeño independiente en los diferentes contextos del niño. De igual manera, la propiocepción va ligada a la coordinación dinámica general, que se dirige sobre todo al control del propio cuerpo para conseguir la precisión y adaptación de los gestos motrices. Gracias a esta los niños logran reconocer sin el apoyo visual sus segmentos corporales y utilizar el tono muscular para ejecutar los patrones motores más importantes en su desarrollo.

La población infantil que presenta desórdenes en la ejecución de sus praxis se caracteriza por la dificultad en el proceso de lectura, escritura y comprensión de conceptos numéricos, así como escasa autonomía en la ejecución de actividades de la vida diaria (AVD) y poco gusto por la 
participación en actividades deportivas; asimismo presentan una tendencia hacia conductas hiperactivas y distráctiles. Estos niños también pueden demostrar defensividad táctil, dificultades en el reconocimiento de lateralidad, el cruce de línea media, la coordinación bimanual y las habilidades viso-perceptuales. (Serna, Torres y Torres, 2017). Dentro de las dificultades en la adquisición de la praxis, algunos autores han identificado disfunciones menores, una de ellas es el trastorno del aprendizaje procedimental (TAP). El tAP se define como un déficit en el aprendizaje motor o una dispraxia gestual, se trata de dificultades de control motor que se han catalogado clásicamente como signos blandos o "menores" de una disfunción cerebral.

Estos niños suelen presentar alteraciones en la motricidad fina y gruesa, dificultad para ejecutar praxias de vestir, poca habilidad en la ejecución de patrones integrales, etc. Otra característica que lo define es la dificultad en el aprendizaje perceptivo-motor, gnosias y praxias; los niños con TAP presentan dificultades para la memoria implícita, que interviene en el aprendizaje de hábitos cognitivos y motores, y para la automatización de la lectoescritura, así como problemas de atención (Crespo-Eguílazy y Narbona, 2009). Asimismo, la calidad de vida de los menores que presentan esta dificultad se ve afectada debido a la desmotivación por la actividad física, acompañada de una disminución de su autoestima. En estos 50 casos, es importante que los maestros creen programas de estimulación para las habilidades motrices, pues estas no mejoran con la maduración, sino que empiezan a afectar el desempeño escolar y el comportamiento (Ruiz, Mata y Moreno, 2007).

\section{Conclusiones}

La integración sensorial se da a partir del trabajo cortical, en el cual el cerebro ejecutivo procesa información de manera innata y adecuada a través del control y la relación de sistemas sensoriales tales como el vestibular, propioceptivo y táctil, lo que permite la interacción eficaz del niño con los diferentes actores de su entorno, consiguiendo así una mayor adaptación personal-social (Ayres, 2005). Cuando esta sincronía de las estructuras cerebrales se altera aparecen retrasos en el desarrollo psicomotor, fallas en la asimilación viso-construccional, limitaciones en las funciones ejecutivas, en procesos psicológicos básicos como la atención, la memoria de trabajo, las habilidades lingüísticas y, por consiguiente, en el aprendizaje e interiorización del código lecto-gráfico.

Se hace evidente entonces, tal como lo define la Federación Mundial de Neurología, que durante la escolaridad se manifiestan síntomas específicos relacionados con la lectoescritura - como es el caso de la dislexia o un trastorno en la capacidad para aprender a leer- a pesar de contar con una instrucción convencional, inteligencia normal y adecuada oportunidad sociocultural: usualmente los niños presentan dificultad para reconocer palabras y discriminar los sonidos del habla; déficit en la asociación grafema-fonema; y fallas en la atención, la 
memoria visual y la estructuración espacial (Serrano y Defior, 2004).

En lo que se refiere a la disgrafía o trastorno de la expresión escrita, que implica problemas grafomotores en cuanto al trazado, la forma y la orientación de las letras, Fuiza y Fernández (2014) describen lo planteado por Rivas y Fernández acerca de las fallas presentes en la coordinación óculo-manual, en la capacidad de organización espaciotemporal, en el equilibrio postural, en la correcta presión-prensión del lápiz y en la velocidad o ritmo escritor. De la misma manera, la disortografía encierra dificultades para la correcta escritura de las palabras, dificultades que no comprometen su trazado o grafía y que se manifiestan en trastornos de tipo lingüístico-perceptivo (omisiones, agregados y sustituciones de fonemas de punto y modo de articulación parecidos, sílabas y/o palabras), visoespacial (inversiones de grafemas de configuración similar pero que se orientan diferente en el espacio, confusión de letras que admiten doble grafía, omisiones y adiciones grafemáticas), visoauditivo (dificultad en la asociación fonema-grafema), de relación con el contenido (fusión o separación incorrecta de sílabas, palabras y frases) y referentes a las reglas ortográficas (mal uso de los signos de puntuación e inadecuada escritura de las palabras).

En síntesis, podría decirse que el proceso de integración sensorial es fundamental en el aprendizaje del código lectoescrito, pues el cerebro permite al individuo registrar sensaciones, reorganizar la información obtenida y consolidar funciones psicológicas superiores; todo ello facilita la asimilación, acomodación y adaptación de manera significativa a las exigencias del ambiente, y sirve a la vez de base para adquirir capacidades intelectuales más complejas.

\section{Referencias}

Ardila, A. y Ostrosky, F. (1991). Diagnóstico del daño cerebral, enfoque neuropsicológico. México: Trillas.

Ardila, A., Roselli, M. y Matute, E. (2005). Neuropsicología de los Trastornos del aprendizaje. Bogotá: Editorial Manual Moderno.

Ayres, J. (2005). La integracion sensorial y el niño. México: Trillas.

Beaudry, I. (2006). Un trastorno en el procesamiento sensorial es frecuente la causa de problemas de aprendizaje,conducta y coordinación motriz en niños. Sociedad de Pediatria de Asturias, 197, 200-203.

Bellefeuille, B. (2006). Un trastorno en el procesamiento sensorial es frecuentemente causa de problemas de aprendizaje, conducta y coordinación motriz en niños. Boletín pediátrico, 46, 200-203.

Caicedo, O.J. (2017). Relación entre las dificultades de integración sensorial, la lectoescritura y aritmética en niños de 7 a 9 años. [Trabajo de grado Máster Universitario en Neuropsicología y Educación]. Popayán: Universidad Internacional de La Rioja. Recuperado de https://reunir.unir.net/ bitstream/handle/123456789/5005/CAICEDO\%20OBANDO\%2C\%20JOSE\%20 YESID.pdf? sequence $=1$ \&isAllowed $=y$

Cancino, A. y Ramos, B. (2007). Descripción del procesamiento sensorialy rendimiento escolar en niños entre 7 y 9 años en un colegio de la región metropolitana. [Tesis de grado]. Santiago de Chile: Universidad de Chile. Recuperado de http://repositorio. uchile.cl/handle/2250/110691 
Crespo-Eguílaz, N. y Narbona, J. (2009). Trastorno de aprendizaje procedimental: características neuropsicológicas. Revista neurología.com, 49(08). Recuperado de https://www.neurologia.com/articulo/2009079

Erazo, O. (2016). Identificación, descripción y relaciones entre la integración sensorial, atención y conducta. Revista Colombiana de Ciencias Sociales, 21-48.

Fodor, E., García, M. y Morán, M. (1997). Todo unmundo de sensaciones. Método de autoayuda parapadres y profesionales aplicado al periodo inicial de la vida. Madrid: Pirámide.

Fuiza, M. y Fernández, M. (2014). Dificultades de aprendizaje y trastornos del desarrollo. Madrid: Pirámide.

Gamboa, K., Mónico, P. y Triana, A. (2018). Relación entre el procesamiento sensorial y el desarrollo de la función ejecutiva de inhibición en niños de 5 a 7 años que presentan trastorno del procesamiento sensorial. [Tesis degrado]. Bogotá: InstituciónUniversitaria Politécnico Grancolombiano.

Gil, R. (2007). Manual de Neurología. [4. ${ }^{\text {E Edi- }}$ ción]. España: Elsevier Masson.

Giner, M. (2008). Psicologíay pedagogía [Blog]. Recuperado de http://psicopedagogias. blogspot.com/2007/07/clav-psicopedagogs.html

Magaña, M. y Ruiz-Lazaro, P. (2018). Trastornos específicos del aprendizaje. Recuperado de https://faros.hsjdbcn.org/ adjuntos/415.2-Ps_inf_trastornos_especificos_aprendizaje.pdf

Moya, D. (2018). La teoría de la integración sensorial. Recuperado de www.seri.es/index.php/component/.../categoty/3-ponencias?...integracionsensorial:2012_Int $\% 20$ Sensorial\%20FINAL\%20Diana\%20 Moya\%20(1).pdf

52 Moya, D. y Matesanz, B. (2010). La teoría de integración sensorial [Ponencia]. Recuperado de https://www.google.com.co/ url?sa $=t \& r c t=j \& q=\& e s r c=s \&$ source $=w e-$ b\&cd $=1$ \&ved $=2$ ahUKEwi6x-7PqZ fkAhVNvFkKHfp0CHkQFjAAegQIAxAC\&url=http\%3A\%2F\%2Fwww.seri. es\%2Findex.php\%2Fcomponent\%2Fphocadownload\%2Fcategory\%2F3-ponencias\%3Fdownload\%3D74\%3Aintegracion-sensorial-metodo-ayres\&usg=AOvVaw3LTUnJZFCDXob-UMr930_g

Pérez, R. (2012). Trastorno de Regulación del Procesamiento sensorial: una contribución para su diagnóstico en la primera infancia. Recuperado de https://www.tdx.cat/ bitstream/handle/10803/117791/rprldel. pdf? sequence $=1$ \&isAllowed $=y$

Ramírez, D. (2016). Características del procesamiento sensorial y su relación con la generación de dificultades de aprendizaje en niños escolares con epilepsia entre los 7 y 10 años, Bogotá (Colombia). [Tesis de maestría en Neurociencias]. Bogotá: Universidad Nacional de Colombia. Recuperado de http://bdigital.unal.edu. co/53090/1/1013606041.2016.pdf

Serna, S., Torres, K. y Torres, M. (2017). Desórdenes en el procesamiento sensorial y el aprendizaje de niños preescolares: Revisión de literatura. Revista Chilena de Terapia Ocupacional, 17, 83-91.

Serrano, F. y Defior, S. (2004). Dislexia en español: estado de la cuestión. Revista Electrónica de Investigación Psicoeducativa y Psicopedagógica, 2(2), 13-34. Recuperado de http://www.redalyc.org/articulo. oa?id=293152879003

Smith, E. y Kosslyn, S. (2008). Procesos cognitivos, modelos y bases neurales. Madrid: Pearson.

Troncoso, P. (2014). Recensión: La eficacia del enfoque de la integración sensorial en la infancia. revistatoq.com, 17-18.

Vidal, M. (2014). Integración sensorial: programa de actuación en educación infantil. [Trabajo de grado]. Universidad Internacional de La Rioja. Recuperadodehttps://reunir.unir.net/ bitstream/handle/123456789/2491/vidal. elortegui.pdf?sequence $=1$ \& \&isAllowed $=y$ 